\title{
Riemannian problems with a fundamental differential system
}

\author{
Rui Albuquerque
}

\begin{abstract}
We introduce the reader to a fundamental exterior differential system of Riemannian geometry which arises naturally with every oriented Riemannian $n+1$ manifold $M$. Such system is related to the well-known metric almost contact structure on the unit tangent sphere bundle $S M$, so we endeavor to include the theory in the field of contact systems. Our EDS is already known in dimensions 2 and 3 , where it was used by $\mathrm{Ph}$. Griffiths in applications to mechanical problems and Lagrangian systems. It is also known in any dimension but just for flat Euclidean space. Having found the Lagrangian forms $\alpha_{i} \in \Omega^{n}, 0 \leq i \leq n$, we are led to the associated functionals $\mathscr{F}_{i}(N)=\int_{N} \alpha_{i}$, on the set of hypersurfaces $N \subset M$, and to their Poincaré-Cartan forms. A particular functional relates to scalar curvature and thus we are confronted with an interesting new equation.
\end{abstract}

\section{Geometric structures and the fundamental differential system}

\subsection{The manifold SM}

Let $M$ be any smooth oriented $n+1$-dimensional Riemannian manifold. Our study is centred on the geometry of the tangent bundle $T M$ as an oriented Riemannian $2 n+$ 2-manifold, endowed with the well-known Sasaki metric. Let $\pi: T M \longrightarrow M$ denote the canonical projection. The vector bundle $V:=\operatorname{ker} \mathrm{d} \pi \simeq \pi^{\star} T M \longrightarrow T M$ agrees fibrewise with the tangent bundle to the fibres of $T M$. Moreover the tangent bundle of $T M$ splits as $T T M=H \oplus V$, where $H$ is a sub-vector bundle depending on $\nabla$, the Levi-Civita connection. Clearly the horizontal sub-bundle $H$ is also isomorphic to $\pi^{*} T M$ through the map $\mathrm{d} \pi$. We thus define an endomorphism

Rui Albuquerque

Dipartimento di Matematica “Giuseppe Peano”, Università degli Studi di Torino, Via Carlo Alberto 10, 10123 Torino, Italy, and Centro de Investigação em Matemática e Aplicações, Universidade de Évora, Portugal, e-mail: rui.albuquerque@unito.it 


$$
B: T T M \longrightarrow T T M
$$

transforming $H$ in $V$ and vanishing on the vertical sub-bundle $V$. This is used by many authors perhaps not giving it so much importance. Partly because one simply recurs to lifts of the same vector on $M$ to either horizontal or vertical parts.

There also exists a connection independent vector field $\xi$ over $T M$ defined by $\xi_{u}=u$, or maybe more precisely $\xi_{u}=\pi^{\star} u, \forall u \in T M$, turning explicit the vertical lift. Henceforth, there exists a unique horizontal $\nabla$-dependent vector field, formally, $B^{\text {ad }} \xi \in H$, such that $B\left(B^{\text {ad }} \xi\right)=\xi$. That field is the geodesic spray of the connection, cf. [17]. One can see easily that $\pi^{\star} \nabla_{w} \xi=w^{v}$, one reason being that $H=\operatorname{ker}\left(\pi^{\star} \nabla . \xi\right)$.

The manifold $T M$ also inherits a linear connection, denoted $\nabla^{*}$, which is just

$$
\pi^{*} \nabla \oplus \pi^{\star} \nabla
$$

preserving the canonical splitting $T T M=H \oplus V \simeq \pi^{*} T M \oplus \pi^{\star} T M$. We observe then that the connecting endomorphism $B$ is parallel for such $\nabla^{*}$. The torsion of $\nabla^{*}$ is given by $\pi^{*} T^{\nabla}(v, w) \oplus \mathscr{R}^{\xi}(v, w), \forall v, w \in T T M$, where the vertical part is $\mathscr{R}^{\xi}(v, w)=R^{\pi^{\star} \nabla}(v, w) \xi=\pi^{\star} R^{\nabla}(v, w) \xi$.

Now we come forward with the metric tensor of $M$. The Sasaki metric $\langle$,$\rangle on T M$ is given naturally by the pull-back of the metric on $M$ both to $H$ and $V$. The parallel mirror morphism $B_{\mid}: H \rightarrow V$ is then metric-preserving. Now $B^{\text {ad }}$ really denotes the adjoint endomorphism of $B$ and the map $J=B-B^{\text {ad }}$ is the Sasaki almost complex structure on $T M$.

Any frame in $H$ extended with its mirror in $V$ clearly determines an orientation on the manifold $T M$. We convention to adopt the order 'first $H$, then $V$ ', which is a relevant issue when $\operatorname{dim} M$ is odd.

Let us suppose $\nabla$ is the Levi-Civita connection and consider the radius 1 tangent sphere bundle

$$
S M=\{u \in T M:\|u\|=1\} .
$$

$\nabla^{*}$ is a metric connection and so, differentiating $\langle\xi, \xi\rangle=1$, we deduce $T S M=\xi^{\perp}$. Since the manifold $T M$ is orientable, $S M$ is also always orientable - the restriction of $\xi$ being a unit outward normal. By the Gram-Schmidt process and the orthogonal group action, for any $u \in S M$ we may find a local horizontal orthonormal frame $e_{0}, e_{1} \ldots, e_{n}$ on a neighbourhood of $u$ in $S M$ and such that $e_{0}=B^{\text {ad }} \xi$ or, equivalently, $e_{0}=u \in H$.

With the dual horizontal coframing, clearly the identity $\pi^{*} \operatorname{vol}_{M}=e^{0} \wedge e^{1} \wedge \cdots \wedge e^{n}$ is satisfied. Adding the mirror subset $\left\{\xi^{b}, e^{n+1}, \ldots, e^{2 n}\right\}$, with $e^{n+i}=e^{i} \circ B^{\text {ad }}, \forall i \geq 1$ (equivalently $\left.e^{n+i}\left(e_{j}\right)=e^{i}\left(e_{j+n}\right)=0, e^{n+i}\left(e_{j+n}\right)=e^{i}\left(e_{j}\right)=\delta_{j}^{i}, \forall i, j\right)$, we find the volume form of $T M$ :

$$
\operatorname{Vol}_{T M}=e^{0} \wedge e^{1} \wedge \cdots \wedge e^{n} \wedge \xi^{b} \wedge e^{n+1} \wedge \cdots \wedge e^{(2 n)}=(-1)^{n+1} \xi^{b} \wedge \operatorname{vol} \wedge \alpha .
$$

We use vol $=\pi^{*} \operatorname{vol}_{M}$; whereas $\alpha$ denotes the $n$-form on $T M$ which is defined as the interior product of $\xi$ with the vertical pull-back of the volume form of $M$. Hence, 
choosing appropriately $\pm \xi$ as unit normal direction, the canonical orientation of the Riemannian submanifold $S M$, given by $\pm \xi\lrcorner \operatorname{Vol}_{T M}$, agrees with $\operatorname{vol} \wedge \alpha=e^{01 \cdots(2 n)}$. A direct orthonormal frame as the one introduced previously is said to be adapted.

\subsection{Further metric properties}

The submanifold $S M$ admits a metric linear connection $\nabla^{\star}$. For any vector fields $y, z$ on $S M$, the covariant derivative $\nabla_{y}^{*} z$ is well-defined and, admitting $y, z$ perpendicular to $\xi$, we just have to add a correction term:

$$
\nabla_{y}^{\star} z=\nabla_{y}^{*} z-\left\langle\nabla_{y}^{*} z, \xi\right\rangle \xi=\nabla_{y}^{*} z+\left\langle y^{v}, z^{v}\right\rangle \xi .
$$

Since $\left\langle\mathscr{R}^{\xi}(y, z), \xi\right\rangle=0$, then a torsion-free connection $D$ is easy to find as $D_{y} z=$ $\nabla_{y}^{\star} z-\frac{1}{2} \mathscr{R}^{\xi}(y, z)$. This connection is most useful for some computations, but ceases to be metric. For the Levi-Civita connection we must add to $D$ another term, $A$, given by:

$$
\left\langle A_{y} z, w\right\rangle=\frac{1}{2}\left(\left\langle\mathscr{R}^{\xi}(y, w), z\right\rangle+\left\langle\mathscr{R}^{\xi}(z, w), y\right\rangle\right) .
$$

Details on metric connections on $S M$ are described in $[4,5]$.

We have found in [9] the conditions for natural maps to become isometries between tangent sphere bundles of different radius, including weighted Sasaki metric and conformal variation of the metric on the base manifold $M$ when $\operatorname{dim} M \geq 3$. Notice the induced horizontal subspaces on $S M$ are not fixed on the same conformal class on $M$. We do not explore here these results with the weights and radius, which are all aloud to be pullbacks of functions on $M$.

Just with the Sasaki metric we have a particular, new result which may catch the readers' attention for those theorems. Consider the constant norm $s>0$ sphere bundle $S_{S} M=s S M$ and let $M=M_{R}^{ \pm}$denote the space-form with metric $g$ of constant sectional curvature $\pm 1 / R^{2}$, where $R>0$.

Proposition 1. Let $g^{S}$ denote the Sasaki metric on the tangent bundle induced from the metric $g$ on $M_{R}^{ \pm}$. Then $\left(S_{S} M_{R}^{ \pm}, g^{S}\right)$ is isometric to $\left(S_{S} M_{1}^{ \pm},\left(R^{2} g\right)^{S}\right)$.

Proof. We use the map $F$ defined in [9, section 2.6] and then apply twice corollary 2.2 from the same article, so the notation now is also from there:

$$
\left(S_{S} M_{R}^{ \pm}, g^{S}\right) \simeq\left(S_{\frac{s}{R}} M_{1}^{ \pm}, g^{R^{2}, R^{2}}\right) \simeq\left(S_{1} M_{1}^{ \pm},\left(R^{2} g\right)^{1, s^{2}}\right) \simeq\left(S_{S} M_{1}^{ \pm},\left(R^{2} g\right)^{S}\right) .
$$

We recall the notation, $g^{f_{1}, f_{2}}=f_{1} \pi^{*} g \oplus f_{2} \pi^{\star} g, g^{S}=g^{1,1}$.

We have also computed in [4] the scalar curvature of the metrics above. For the weighted metric with $f_{1}, f_{2}$ constant, we have

$$
\operatorname{Scal}_{\left(S_{s} M_{R}, g f_{1}, f_{2}\right)}= \pm \frac{n(n+1)}{f_{1} R^{2}}-\frac{f_{2}}{4 f_{1}^{2}} \frac{s^{2}}{R^{4}} 2 n+\frac{(n-1) n}{f_{2} s^{2}}
$$


which is a positive (negative) constant for small (large) $s$, although we do not have an Einstein metric. The value of these results from $[4,9]$ has only recently been understood. Of course it is fun to verify the isometric invariance of our formulas.

\subsection{The contact structure}

We denote by $\theta$ the 1 -form on $S M$ defined by

$$
\theta=\left(B^{\mathrm{ad}} \xi\right)^{b}=\langle\xi, B \cdot\rangle=e^{0}
$$

Y. Tashiro discovered in the 1960's that $\theta$ defines a metric contact structure, cf. [10]. In our adapted frame we find $\mathrm{d} \theta=e^{(1+n) 1}+\cdots+e^{(2 n) n}$. In other words, $\forall v, w \in$ $T S M, \mathrm{~d} \theta(v, w)=\langle v, B w\rangle-\langle w, B v\rangle$.

Now we present the set of natural $n$-forms $\alpha_{0}, \alpha_{1}, \ldots, \alpha_{n}$ existing always on $S M$. Together with $\theta$ they consist of the fundamental differential system we have announced. But we begin with the low dimension cases before a general definition.

In case $n=1$ we have a global coframing of $S M$ with $\theta$ and two 1 -forms $\alpha_{0}=e^{2}$ and $\alpha_{1}=e^{1}$, which are global forms. The following formulas were probably already known (to Cartan?), where $k$ denotes the Gauss curvature of $M$ :

$$
\mathrm{d} \theta=\alpha_{0} \wedge \alpha_{1} \quad \mathrm{~d} \alpha_{0}=k \alpha_{1} \wedge \theta \quad \mathrm{d} \alpha_{1}=\theta \wedge \alpha_{0} .
$$

For the case $n=2, \alpha_{0}=e^{34}, \alpha_{1}=e^{14}+e^{32}, \alpha_{2}=e^{12}$, or the case $n=3, \alpha_{0}=$ $e^{456}, \alpha_{1}=e^{156}+e^{264}+e^{345}, \alpha_{2}=e^{126}+e^{234}+e^{315}, \alpha_{3}=e^{123}$, we do not have any special example or easier way of computing the exterior derivatives other than that which we use in [8] with the connections $\nabla^{*}, D$ above - except in case $n=3$ and flat metric coordinates, as shown in [3], because the 3-sphere is parallelizable and so we may explicit an adapted frame (just as with $n=1$ ).

Finally we define the $n+1$ natural $n$-forms on $S M$. First, for $0 \leq i \leq n$, let

$$
n_{i}=\frac{1}{i !(n-i) !}
$$

Continuing with the adapted frame introduced earlier, we then define:

$$
\left.\alpha_{0}=\alpha=\xi\right\lrcorner\left(\pi^{\star} \operatorname{vol}_{M}\right)=e^{(n+1)} \wedge \cdots \wedge e^{(2 n)}
$$

where $\pi^{\star} \mathrm{vol}_{M}$ is the vertical pull-back of the volume form of $M$. Now for each $i$ we write, $\forall v_{1}, \ldots, v_{n} \in T S M$,

$$
\alpha_{i}\left(v_{1}, \ldots, v_{n}\right)=n_{i} \sum_{\sigma \in S_{n}} \operatorname{sg}(\sigma) \alpha\left(B v_{\sigma_{1}}, \ldots, B v_{\sigma_{i}}, v_{\sigma_{i+1}}, \ldots, v_{\sigma_{n}}\right) .
$$

We remark that $\alpha_{n}=e^{1 \ldots n}$, which justifies the introduction of the weight $n_{i}$. For convenience we define $\alpha_{-1}=\alpha_{n+1}=0$. 
Only a scarce number of references have used the exterior differential system of $\theta$ and the $\alpha_{i}$, yet not rising them to the level of a field of study. It seems the $n$-forms have only been considered as an auxiliary tool in the solution of very few mechanical systems problems. First for 2 or 3 dimensional base space in Ph. Griffiths' book [13]. Then in [11, p.152] with emphasis on a 3-dimensional metric and an algebraic problem. The same being true regarding later articles in [14], as well as in [15].

Regarding the $n$-dimensional case, we suppose to be correct in saying it appears for the first time, though only for the Euclidean base space, in [12, p. 32]. To the best of our knowledge, the definition in full generality (11) is introduced first by the author in [8].

Our differential system is original for we do not have any other reference for the following formulas deduced in [8]. On a manifold with constant sectional curvature $k$ we have

$$
\begin{aligned}
& \mathrm{d} \alpha_{0}=\theta \wedge\left(\quad-k \alpha_{1}\right) \\
& \mathrm{d} \alpha_{1}=\theta \wedge\left(\quad n \alpha_{0}-2 k \alpha_{2}\right) \\
& \mathrm{d} \alpha_{2}=\theta \wedge\left((n-1) \alpha_{1}-3 k \alpha_{3}\right) \\
& \mathrm{d} \alpha_{n-1}=\theta \wedge\left(2 \alpha_{n-2}-n k \alpha_{n}\right) \\
& \mathrm{d} \alpha_{n}=\theta \wedge \alpha_{n-1}
\end{aligned}
$$

or simply $\mathrm{d} \alpha_{i}=\theta \wedge\left((n-i+1) \alpha_{i-1}-k(i+1) \alpha_{i+1}\right), \forall i=0, \ldots, n$. The particular case of formula (12) with sectional curvature $k=0$ is already known, as we referred.

\subsection{Some structural relations}

The proofs of the following are quite easy, cf. [8]. For any $0 \leq i \leq n$ we have:

$$
\begin{aligned}
*(\mathrm{~d} \theta)^{i} & =(-1)^{\frac{n(n+1)}{2}} \frac{i !}{(n-i) !} \theta \wedge(\mathrm{d} \theta)^{n-i} \\
* \alpha_{i} & =(-1)^{i} \theta \wedge \alpha_{n-i} .
\end{aligned}
$$

Also $\alpha_{i} \wedge \mathrm{d} \theta=0$ and $\alpha_{i} \wedge \alpha_{j}=0, \forall j \neq n-i$. Of course $*$ denotes the Hodge staroperator on $S M$, which satisfies $* *=1$ on $\Lambda_{S M}^{*}$. In our notation,

$$
R_{i j k l}=\left\langle\nabla_{e_{i}} \nabla_{e_{j}} e_{k}-\nabla_{e_{j}} \nabla_{e_{i}} e_{k}-\nabla_{\left[e_{i}, e_{j}\right]} e_{k}, e_{l}\right\rangle .
$$

Theorem 1 (1st-order structure equations, [8]). We have

$$
\mathrm{d} \alpha_{i}=(n-i+1) \theta \wedge \alpha_{i-1}+\mathscr{R}^{\xi} \alpha_{i}
$$

where 


$$
\left.\mathscr{R}^{\xi} \alpha_{i}=\sum_{0 \leq j<q \leq n} \sum_{p=1}^{n} R_{j q 0 p} e^{j q} \wedge e_{p+n}\right\lrcorner \alpha_{i} .
$$

This theorem is proved with the tools of connection theory introduced in the first section. We do not have any other method which could ease the computations.

Defining $r=\operatorname{Ric}(\xi, \xi)=\sum_{j=1}^{n} R_{j 00 j}$ as a smooth function on $S M$ determined by the Ricci curvature of $M$, we have after computations ([8])

$$
\mathrm{d} \alpha_{n}=\theta \wedge \alpha_{n-1} \quad \mathrm{~d} \alpha_{n-1}=2 \theta \wedge \alpha_{n-2}-r \mathrm{vol},
$$

i.e. $\mathscr{R}^{\xi} \alpha_{n}=0$ and $\mathscr{R}^{\xi} \alpha_{n-1}=-r \theta \wedge \alpha_{n}$. Then clearly

$$
\mathrm{d}\left(\mathscr{R}^{\xi} \alpha_{i}\right)=(n-i+1) \theta \wedge \mathscr{R}^{\xi} \alpha_{i-1} \quad \mathrm{~d} \theta \wedge \mathscr{R}^{\xi} \alpha_{i}=0 .
$$

Proposition 2. The differential forms $\theta, \alpha_{0}$ and $\alpha_{1}$ are always coclosed. Moreover, for all $0 \leq i \leq n$,

$$
\mathrm{d}\left(i * \alpha_{i}+(-1)^{i} \mathscr{R}^{\xi} \alpha_{n-i+1}\right)=0 .
$$

Proof. One just applies (13) and (18):

$$
\mathrm{d} i * \alpha_{i}=i(-1)^{i+1} \theta \wedge \mathrm{d} \alpha_{n-i}=i(-1)^{i+1} \theta \wedge \mathscr{R}^{\xi} \alpha_{n-i}=(-1)^{i+1} \mathrm{~d}\left(\mathscr{R}^{\xi} \alpha_{n-i+1}\right)
$$

Clearly, $\mathscr{R}^{\xi} \alpha_{n+1}=0$ and it is true $\mathrm{d} * \alpha_{n}=\mathrm{dvol}=0$.

No further assumptions on $M$ are required, so we believe there are good reasons to refer to the d-closed differential ideal $\mathscr{I}=\operatorname{span}\left\{\theta, \alpha_{0}, \ldots, \alpha_{n}\right\}$ as a fundamental object of any oriented Riemannian $n+1$-manifold.

It is quite interesting to consider the case of constant sectional curvature $k$ in any dimension. The Riemann curvature tensor is $R_{i j p q}=k\left(\delta_{i q} \delta_{j p}-\delta_{i p} \delta_{j q}\right)$, so one may prove that $\mathscr{R}^{\xi} \alpha_{i}=-k(i+1) \theta \wedge \alpha_{i+1}$, cf. (12).

\subsection{Gwistor space and problems for calibrated geometries}

The author's discovery of the exterior differential system $\mathscr{I}$ came after and with that of a natural $\mathrm{G}_{2}$ structure on $S M$ for $M$ of dimension 4.

In $[1,2]$ it is proved that the total space of the radius 1 tangent sphere bundle $S M \longrightarrow M$ of any given oriented Riemannian 4-manifold $M$ carries a natural $\mathrm{G}_{2}$ structure. The space is now called $\mathrm{G}_{2}$-twistor or gwistor space. Its fundamental structure 3 -form is

$$
\phi_{1}=\theta \wedge \mathrm{d} \theta+\alpha_{2}-\alpha_{0} .
$$

Gwistor space is being studied as a subject of its own importance. It has had several developments in $[1,2,3,6,7]$ in relation with $\mathrm{G}_{2}$ geometry. We know that $\phi_{1}$ is never closed and it is coclosed if and only if $M$ is Einstein. The $\mathrm{G}_{2}$ structure $\phi_{2}=$ $\theta \wedge \mathrm{d} \theta+\alpha_{3}-\alpha_{1}$ is more restrictive. There is a circle of $\mathrm{G}_{2}$ structures on $S M$ within $\phi_{1}$ and $\phi_{2}$ compatible with the Sasaki metric. 
An important open problem in linear algebra is to find the conditions for which a linear combination $\varphi=\sum_{i=0}^{n} b_{i} \alpha_{i}+c \theta^{\varepsilon} \wedge(\mathrm{d} \theta)^{\left[\frac{n}{2}\right]}$, with $b_{i}, c \in \mathrm{C}_{S M}^{\infty}, \varepsilon=0,1$, becomes a calibration. Recall a calibration is a closed $p$-form $\varphi$ such that $\varphi_{\mid V} \leq \operatorname{vol}_{V}$ for every oriented tangent $p$-plane $V$, cf. [16]. One expects all $b_{i}, c$ to be constant, yet we are unable to eliminate other possibility.

For even $n=\operatorname{dim} M-1$ we have an obvious $\varphi$ of degree $n$. For $n=1$ the question may be solved easily recurring to (8). For $n=2$ and 3 we have a complete linear algebra classification of the calibrations in [16, Theorems 4.3.2 and 4.3.4]. In case $n=3$, we recover gwistor space.

The following result is quite interesting. Let $\rho=\xi\lrcorner \pi^{\star}$ Ric, the vertical lift of the Ricci tensor. With an adapted frame, we deduce

$$
\rho=\sum_{a, b=1}^{n} R_{a b 0 a} e^{b+n} .
$$

We have the following theorem giving a reduction of the degree of a differential equation.

Theorem 2 ([8]). In any dimension we have $\mathrm{d} * \alpha_{2}=\rho \wedge$ vol. Henceforth, the metric on $M$ is Einstein if and only if $\delta \alpha_{2}=0$.

\section{Geometric applications}

\subsection{Recalling Euler-Lagrange systems}

We wish to study the Euler-Lagrange system $(S M, \theta, \varphi)$, where $\varphi$ is a calibration, in applications to Riemannian geometry. We start further above recalling the theory of contact systems from [12]. In this section we assume $(S, \theta)$ is any given contact manifold, not necessarily metric, of dimension $2 n+1$.

The contact differential ideal $\mathscr{I}$ is defined as the d-closed ideal generated by $\theta \in \Omega_{S}^{1}$. A generalisation of the famous Darboux Theorem assures that locally $S$ is the 1-jet manifold $J^{1}\left(\mathbb{R}^{n}\right)$ of Euclidean flat space, with (Pfaff) coordinates $\left(z, x^{i}, p_{i}\right)$ and contact form $\theta=\mathrm{d} z-\sum_{i=1}^{n} p_{i} \mathrm{~d} x^{i}$. The submanifold $N$ given by $z=0, p_{i}=0$ satisfies $\boldsymbol{\theta}_{\mid N}=0$. That is also the case with any submanifold $\left\{\left(z(x), x^{i}, \partial_{i} z\right)\right\}$ where $z$ is a $\mathrm{C}^{1}$ function on the $x^{i}$.

An integral submanifold of $S$ consists of a submanifold $N$ together with an immersion $f: N \rightarrow S$ such that $f^{*} \theta=0$. Then of course $f^{*} \mathscr{I}=0$. A Legendre submanifold is a $C^{1}$-differentiable integral $n$-dimensional submanifold $N$. The Legendre submanifolds which appear as the graph of a function on $N$ in the Pfaff coordinates are called transverse. Equivalently, $N$ is transverse if and only if $f^{*}\left(\mathrm{~d} x^{1} \wedge \cdots \wedge \mathrm{d} x^{n}\right) \neq 0$. 
Any form $\Lambda \in \Omega_{S}^{n}$ is called a Lagrangian. An equivalence relation is immediately associated with equivalence class $\Lambda+\mathscr{I}^{n}+\mathrm{d} \Omega^{n-1}$, where $\Lambda$ is a representative and $\mathscr{I}^{n}=\mathscr{I} \cap \Omega^{n}$.

An algebraic identity deduced in [12] carries over to the whole contact manifold as:

$$
\mathscr{I}^{k}=\Omega^{k}, \quad \forall k>n .
$$

Hence there exist two forms $\alpha, \beta$ on $S$ such that $\mathrm{d} \Lambda=\theta \wedge \alpha+\mathrm{d} \theta \wedge \beta=\theta \wedge(\alpha+$ $\mathrm{d} \beta)+\mathrm{d}(\theta \wedge \beta)$. By [12, Theorem 1.1] there exists a unique global exact form $\Pi$ such that $\Pi \wedge \theta=0$ and $\Pi \equiv \mathrm{d} \Lambda$ in $\bar{H}^{n+1}\left(\Omega^{*} / \mathscr{I}, \mathrm{d}\right)$. The Poincaré-Cartan form is $\Pi=\mathrm{d}(\Lambda-\theta \wedge \beta)=\theta \wedge(\alpha+\mathrm{d} \beta)$. The form $\Psi=\alpha+\mathrm{d} \beta$ turns out to be of great importance.

Now one wishes to find the critical points of a functional on the set of smooth, compact Legendre submanifolds $N \hookrightarrow S$, possibly with boundary, defined by:

$$
\mathscr{F}_{\Lambda}(N)=\int_{N} f^{*} \Lambda
$$

Note that $\Lambda$ clearly induces the same functional on its class for Legendre submanifolds without boundary.

Suppose we have a variation of Legendre submanifolds with fixed boundary, i.e. suppose there is a curve of smooth maps $f_{t}: N \rightarrow S$ which defines a Legendre submanifold $N_{t}$ for each $t$ and $\partial\left(N_{t}\right)=\partial\left(N_{0}\right)$. Differentiating $\mathscr{F}_{\Lambda}\left(N_{t}\right)$, cf. [12], leads to the conclusion that

$$
\frac{\mathrm{d}}{\mathrm{d} t}_{\mid t=0} \mathscr{F}_{\Lambda}\left(N_{t}\right)=0 \quad \text { if and only if } \quad f^{*} \Psi=0 .
$$

A Legendre submanifold satisfying (24) is called a stationary Legendre submanifold. The exterior differential system algebraically generated by $\theta, \mathrm{d} \theta, \Psi$ is called the Euler-Lagrange system of $(S, \theta, \Lambda)$; its Poincaré-Cartan form $\Pi$ is said to be non-degenerate if it has no other degree 1 factors besides the multiples of $\theta$.

In order to determine conditions on the stationary submanifolds of $\mathscr{F}_{\Lambda}$ one proceeds as follows: find the Poincaré-Cartan $n$-form, transform it into the product $\theta \wedge \Psi$ and then do the analysis of $f^{*} \Psi=0$, i.e. study the Euler-Lagrange equation.

\subsection{On the unit tangent sphere bundle}

Let us admit again an oriented $n+1$-dimensional Riemannian manifold $M$ together with its unit tangent sphere bundle $S M \stackrel{\pi}{\longrightarrow} M$. Now we let $f: N \rightarrow M$ be a compact oriented isometric immersed hypersurface.

Then we have also a smooth lift $\hat{f}: N \rightarrow S M$ of $f$, the unique unit normal $v \in$ $T_{f(x)} M$ chosen according to the orientations of $N$ and $M$. Note that $\hat{f}$ is also defined on $\partial N$. It is easy to see that we have the decomposition into horizontal plus vertical: 


$$
\mathrm{d} \hat{f}(w)=(\mathrm{d} f(w))^{h}+\left(f^{*} \nabla\right)_{w} f^{*} v, \quad \forall w \in T_{x} N .
$$

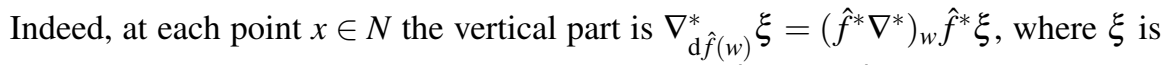
the canonical vertical vector field on $S M$. Clearly, $\left(\hat{f}^{*} \xi\right)_{x}=\hat{f}(x)=v_{f(x)}=\left(f^{*} v\right)_{x}$ and $\hat{f}^{*} \pi^{*}=f^{*}$. By definition of $\hat{f}$ we clearly have that $\hat{f}: N \longrightarrow S M$ defines a Legendre submanifold of the natural contact structure, $\hat{f}^{*} \theta=0$, and that it is a transverse submanifold.

A smooth Legendre submanifold $Y$ is locally the lift $N \rightarrow Y \subset S M$ of an oriented smooth $n$-submanifold $N \hookrightarrow M$ if and only if $e^{1 \cdots n}{ }_{\mid Y} \neq 0$, i.e. precisely when $Y$ is transverse. We are thus going to assume throughout such open condition on submanifolds, defined by the top differential form: $\alpha_{n \mid Y} \neq 0$.

Let us consider an adapted direct orthonormal coframe $e^{0}, e^{1}, \ldots, e^{n}, e^{n+1}, \ldots, e^{2 n}$ locally defined on $S M$. Then it may not be tangent to $N \subset S M$. Yet we have also a direct orthonormal coframe $e^{1}, \ldots, e^{n}$ for $N$ (we use the same letters for the pullback). Now, from (25), for any $1 \leq j \leq n$ we have

$$
\hat{f}^{*} e^{j}=e^{j} \quad \text { and } \quad \hat{f}^{*} e^{j+n}=-\sum_{k=1}^{n} A_{k}^{j} e^{k}
$$

with $A$ the second fundamental form of $N$. We recall, $A=-\nabla v: T N \rightarrow T N$ is a symmetric endomorphism; the associated tensor $H=\frac{1}{n}(\operatorname{Tr} A) v$ is the mean curvature vector field.

We now consider the $n$-forms $\alpha_{i}$, which give in their own right interesting Lagrangian systems on the contact manifold $S M$. We wish to study the functionals $\mathscr{F}_{i}=\mathscr{F}_{\alpha_{i}}$ on the set of compact immersed hypersurfaces of $M$ with fixed boundary.

Let $\sigma_{i}(A)$ denote the elementary symmetric polynomial of degree $i$ in the eigenvalues $\lambda_{1}, \ldots, \lambda_{n}$ of $A$. Then we have that

$$
\hat{f}^{*} \alpha_{n-i}=(-1)^{i} \sigma_{i}(A) \operatorname{vol}_{N} .
$$

We do not know a more simple proof for the following result (only for Euclidean base space it is in [12] with the same method), than by using (24) on $\mathscr{F}_{n}$ and the Poincaré-Cartan form given by $\mathrm{d} \alpha_{n}=\theta \wedge \alpha_{n-1}$.

Theorem 3 (Classical theorem, [8]). Let $N$ be a compact isometrically immersed hypersurface in the Riemannian manifold $M$. Then, $\forall v \in \Gamma_{0}\left(N, f^{*} T M\right)$,

$$
\delta \operatorname{vol}(N)(v)=-\int_{N} n\langle v, H\rangle \operatorname{vol}_{N} .
$$

In particular, $N$ is minimal for the volume functional within all compact hypersurfaces with fixed boundary $\partial N$ if and only if $H=0$.

As used previously, one deduces $\hat{f}^{*} \alpha_{n-1}=-n\langle H, v\rangle \operatorname{vol}_{N}=-n\|H\| \operatorname{vol}_{N}$, hence the functional $\mathscr{F}_{n-1}$ corresponds with

$$
\mathscr{F}_{n-1}(N)=-n \int_{N}\|H\| \operatorname{vol}_{N}
$$


i.e. the integral of the mean curvature on immersed submanifolds $N \subset M$.

Theorem 4 ([8]). Suppose the Riemannian manifold $M$ has dimension $n+1>2$. Then a compact isometric immersed hypersurface $f: N \rightarrow M$ with fixed boundary is stationary for the mean curvature functional $\mathscr{F}_{n-1}$ if and only if

$$
\mathrm{Scal}^{N}=\mathrm{Scal}^{M}-r_{v}
$$

where $r_{v}=\operatorname{Ric}(v, v)$ is induced from the Ricci tensor of $M$ and Scal denotes scalar curvature functions.

In particular, if $M$ is an Einstein manifold, say where Ric $=c g$ with $c$ a constant, then $N$ has stationary mean curvature volume if and only if $N$ has constant scalar curvature $\mathrm{Scal}^{N}=n c$.

For an Einstein metric on the ambient manifold $M$, a formula in the last proof shows that $\mathscr{F}_{n-2}$ leads to an Euler-Lagrange equation essentially on the scalar curvature of $N$.

Theorem 5 ([8]). Let $M$ be a Riemannian manifold of dimension $n+1>2$ and constant sectional curvature $k$. Then a compact hypersurface $N$ is a critical point of the scalar curvature functional $\int_{N} \mathrm{Scal}^{N} \mathrm{vol}_{N}$ with fixed boundary if and only if the eigenvalues $\lambda_{1}, \ldots, \lambda_{n}$ of A satisfy (assume $\lambda_{3}=0$ for $n=2$ )

$$
6 \sum_{j_{1}<j_{2}<j_{3}} \lambda_{j_{1}} \lambda_{j_{2}} \lambda_{j_{3}}+k(n-1)(n-2)\left(\lambda_{1}+\cdots+\lambda_{n}\right)=0 .
$$

In other words, $6 \sigma_{3}(A)+k n(n-1)(n-2)\|H\|=0$.

The case $n=2$ is always satisfied and invariant of the ambient manifold - that is partly the theorem of Gauss-Bonnet.

Acknowledgements The research leading to these results has received funding from the People Programme (Marie Curie Actions) of the European Union's Seventh Framework Programme (FP7/2007-2013) under REA grant agreement n PIEF-GA-2012-332209.

\section{References}

1. Albuquerque, R., Salavessa, I.: The $\mathrm{G}_{2}$ sphere of a 4-manifold. Monatsh. Mathematik 158 (4), 335-348 (2009)

2. Albuquerque, R., Salavessa, I.: Erratum to: $\mathrm{The}_{2} \mathrm{G}_{2}$ sphere of a 4-manifold. Monatsh. Mathematik 160 (1), 109-110 (2010)

3. Albuquerque, R.: On the $\mathrm{G}_{2}$ bundle of a Riemannian 4-manifold. J. Geom. Phys. 60, 924-939 (2010)

4. Albuquerque, R.: Curvatures of weighted metrics on tangent sphere bundles. Riv. Mat. Univ. Parma 2, 299-313 (2011)

5. Albuquerque, R.: Weighted metrics on tangent sphere bundles. Quart. J. Math. 63 (2), 259$273(2012)$ 
6. Albuquerque, R.: On the characteristic connection of gwistor space. Central European J. Math. 11(1) (2013), 149-160

7. Albuquerque, R.: Variations of gwistor space. Portugaliae Mathematica 70 (2), 145-160 (2013)

8. Albuquerque, R.: A fundamental differential system of Riemannian geometry. http://arxiv.org/abs/1112.3213

9. Albuquerque, R.: Homotheties and topology of tangent sphere bundles. Journal of Geometry (2014) doi: http://dx.doi.org/10.1007/s00022-014-0210-x

10. Blair, D.: Riemannian geometry of contact and symplectic manifolds. Birkhaüser Boston Inc., 203, Progress in Mathematics, Boston, MA (2002)

11. Bryant, R., Chern, S. S., Gardner, R., Goldschmidt, H., Griffiths, Ph.: Exterior differential systems. MSRI Publications, Springer Verlag, 18, New York (1991)

12. Bryant, R., Griffiths, Ph., Grossman, D.: Exterior differential systems and Euler-Lagrange partial differential equations. University of Chicago Press, 2003

13. Griffiths, Ph.: Exterior differential systems and the calculus of variations. Birkhaïser, Progress in Mathematics, 25,Boston, Basel, Stuttgart (1983)

14. Griffiths, Ph.: Selected Works of Phillip A. Griffiths with Commentary. American Mathematical Society, Part 4 "Differential Systems", Providence, R.I. (2003)

15. Ivey, Th., Landsberg, J.: Cartan for beginners: differential geometry via moving frames and exterior differential systems. American Mathematical Society, 61, Graduate Studies in Mathematics, Providence, RI (2003)

16. Joyce, D.: Riemannian Holonomy Groups and Calibrated Geometry. Oxford University Press, Oxford Graduate Texts in Mathematics (2009)

17. Sakai, T.: Riemannian Geometry. vol. 149 of Transl. Math. Mono., AMS (1996).

18. Sasaki, S.: On the differential geometry of tangent bundles of Riemannian manifolds. Tôhoku Math. J. 10, 338-354 (1958) 